\title{
APACHE-AF: Apixaban versus antiplatelet drugs or no antithrombotic treatment after anticoagulation-associated intracerebral haemorrhage in patients with atrial fibrillation. A randomised phase II clinical trial.
}

FHBM Schreuder ${ }^{1}$, KM van Nieuwenhuizen ${ }^{2}$, A Algra², LJ Kappelle², GJE Rinkel², H Kerkhoff ${ }^{3}$, GJ Luijckx ${ }^{4}$ J Hofmeijer ${ }^{5}$, HP

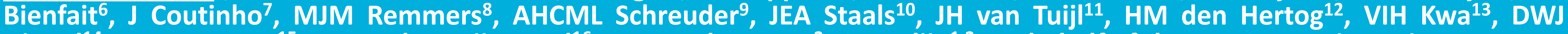
Dippel14, MJH Wermer ${ }^{15}$, IR van den Wijngaard ${ }^{16}$, HB van der Worp ${ }^{2}$, CJM Klijn ${ }^{1,2}$; on behalf of the APACHE-AF investigators.

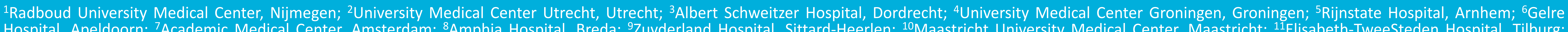

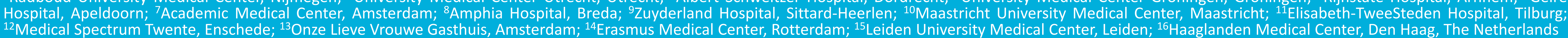

\section{Background}

There is a lack of evidence on the optimal prevention of ischaemic stroke in patients with atrial fibrillation and a recent intracerebral haemorrhage $(\mathrm{ICH})$ during treatment with oral anticoagulation. Patients are currently treated with:

- oral anticoagulants

- antiplatelet drugs, or

- no antithrombotic treatment.

Treatment with a direct oral anticoagulant like apixaban might be an attractive alternative in terms of a lower risk of recurrent $\mathrm{ICH}$ than with a vitamin-K antagonist, while at the same time being effective for the prevention of ischaemic stroke.

\section{Objective}

To obtain reliable estimates of the rates of vascular death or nonfatal stroke in patients with atrial fibrillation and a recent anticoagulation-associated $\mathrm{ICH}$ who are treated with apixaban versus those who are not treated with oral anticoagulation.

\section{Study design}

Multi-centre, phase II, randomised, open-label clinical trial with blinded outcome assessment.

\section{Study population}

100 adults with a history of atrial fibrillation and a recent intracerebral haemorrhage during treatment with oral anticoagulation in whom clinical equipoise exists on the optimal stroke prevention therapy.
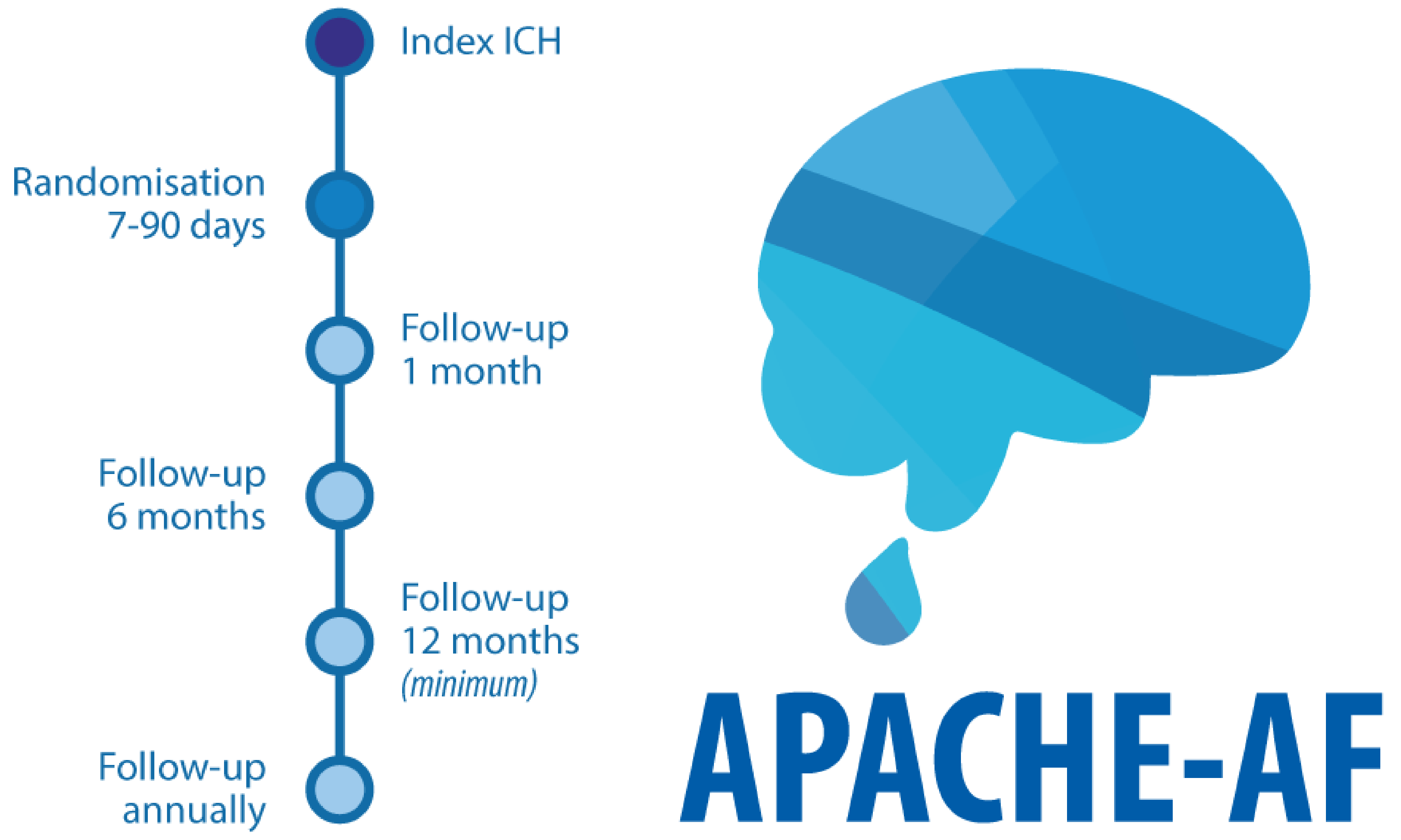

Fig. 1 | Trial flowchart

\section{Trial status (May 2018)}

16 sites recruiting the Netherlands

Fig. 2 | Participating sites

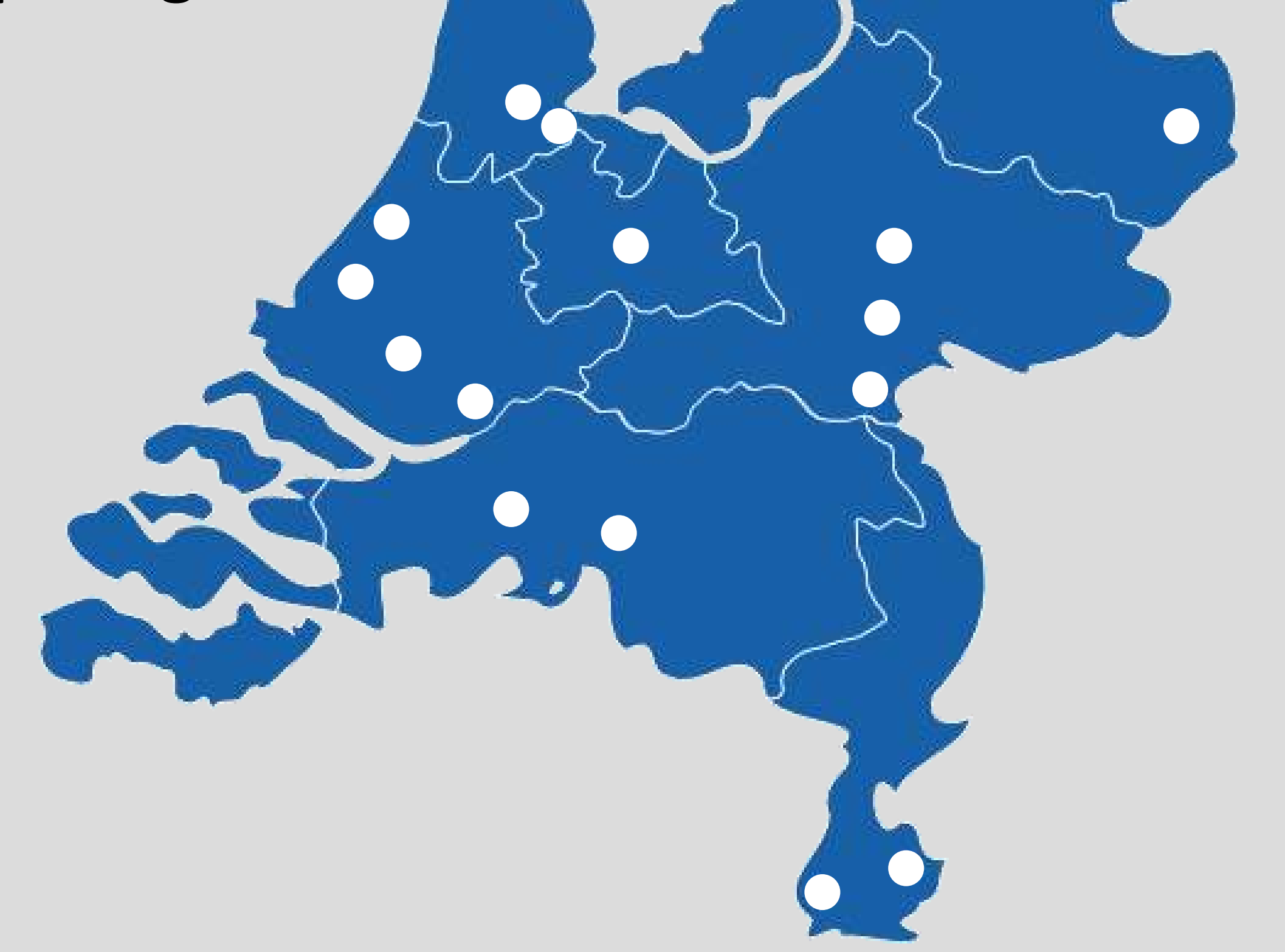

46 patients randomized, with a mean age of 77.5 years (SD

7.5 years), 25 males ( $54 \%)$

17 patients with lobar and 29 with non-lobar ICH

66.4 patient-years of follow-up

\section{Intervention}

Patients will be randomised between 7 and 90 days after the index haemorrhage to

1) apixaban $5 \mathrm{mg}$ twice daily or

2) any antiplatelet drug or no antithrombotic treatment,

at the discretion of the treating physician.

\section{Primary outcome measure}

Vascular death or non-fatal stroke during follow-up.

\section{Sample size}

Ten primary outcome events in 100 patient-years of followup will yield a $95 \%$ confidence interval of 4.9 to 17.6 .

\section{Funding}

Prof. C.J.M. Klijn is supported by a clinical established investigator grant of the Dutch Heart Foundation (grant number 2012T077) and an ASPASIA grant from ZonMw (grant number 015008048). Dr. H.B. van der Worp is supported by a clinical established investigator grant of the Dutch Heart Foundation (grant number 2010T075). 